\title{
HOMOTOPY ASSOCIATIVITY OF $H$-SPACES. I
}

\author{
BY \\ JAMES DILLON STASHEFF(1)
}

1. Introduction. The concept of an $H$-space arose as a generalization of that of a topological group. The essential feature which is retained is a continuous multiplication with a unit. There is a significant class of spaces which are $H$-spaces but not topological groups. Some of the techniques which apply to topological groups can be applied to $H$-spaces, but not all. From the point of view of homotopy theory, it is not the existence of a continuous inverse which is the important distinguishing feature $[6 ; 15]$, but rather the associativity of the multiplication.

For example, if we regard $S^{0}, S^{1}, S^{3}$ and $S^{7}$ as the real, complex, quaternionic and Cayley numbers of unit norm, these spaces possess continuous multiplications, which in the first three cases are associative. Now it is possible to define real, complex and quaternionic projective spaces of arbitrarily large dimension, but this is not possible for the Cayley numbers. From the point of view of homotopy theory, we can investigate the "mechanism" which relates the associativity of the multiplication to the possible existence of projective spaces.

First we consider the construction of the classical projective space as generalized by Milnor [8] for an arbitrary topological group (and further generalized by Dold and Lashof [3] for an arbitrary associative $H$-space). Given a topological group $G$, Milnor constructs fibre bundles $p_{i}: E_{i} \rightarrow B_{i}$ with fibre $G$, the total space $E_{i}$ being the $i$-fold join of $G$ with itself. If $G=S^{d-1}, d=1,2,4$, this gives the standard fibring of $S^{i d-1}$ onto the corresponding projective space of dimension $i-1$. In the case of the Cayley numbers, only the fibrings of $S^{7}$ onto a point and of $S^{\mathbf{1 5}}$ onto $S^{\mathbf{8}}$ can be constructed. It seems reasonable to ask whether something weaker than associativity might permit more but not all of these fibrings to be constructed. Sugawara [14] has shown that a variant of Milnor's construction can be carried one step further than for an arbitrary $H$-space if the multiplication is at least homotopy associative; that is, if $m: X \times X \rightarrow X$ is the multiplication then the two maps of $X \times X \times X$ into $X$ given by the two ways of associating are homotopic, i.e., the diagram

Presented to the Society, January 22, 1959 under the title On homotopy associativity of $H$-spaces and April 25, 1959 under the title On higher homotopy associativity; received by the editors March 8, 1962.

(1) The author is a C.L.E. Moore Instructor. This research was supported in part by an NSF predoctoral fellowship, in part by the Marshall Aid Commemorative Commission of the United Kingdom, and in part by the United States Air Force under contract No. AF-49(638)-42, monitored by the Air Force Office of Scientific Research of the Air Force Research and Development Command. 


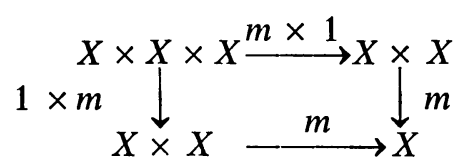

is homotopy commutative. What should the next step be?

From the work of Sugawara and Dold-Lashof, it is clear that a fibre bundle is too restrictive a concept. The features of the Milnor construction which it is important to retain are embodied in the following definition, but first let us stipulate that all spaces we consider will be of the homotopy type of countable $\mathrm{CW}$-complexes with base points and all maps and homotopies will respect base points.

Definition 1. An $A_{n}$-structure on a space $X$ consists of an $n$-tuple of maps

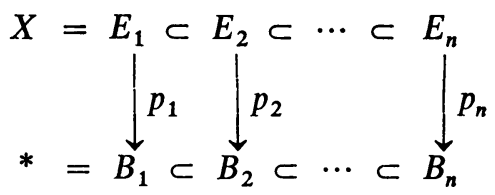

such that $p_{i *}: \pi_{q}\left(E_{i}, X\right) \rightarrow \pi_{q}\left(B_{i}\right)$ is an isomorphism for all $q$, together with a contracting homotopy $h: C E_{n-1} \rightarrow E_{n}$ such that $h\left(C E_{i-1}\right) \subset E_{i}$.

The Milnor or Dold and Lashof construction shows that there are spaces which admit $A_{n}$-structures for all values of $n$.

(For the purposes of homotopy theory, we can think of $X \rightarrow E_{i} \stackrel{p_{i}}{\rightarrow} B_{i}$ as a fibring in the light of

Proposition 2. Given $X \subset E$ and a map $p:(E, X) \rightarrow\left(B,{ }^{*}\right)$ such that $p_{*}: \pi_{q}(E, X) \rightarrow \pi_{q}(B)$ is an isomorphism for all $q$, there exists a homotopy equivalent fibring $F \rightarrow \bar{E} \stackrel{\vec{p}}{\rightarrow} B$ such that $F$ has the homotopy type of $X$.

Proof. Any map is equivalent to a fibring so that we have

$$
\underset{B}{E \rightarrow \bar{E}}
$$

$\left[11\right.$, Lemma 13]. Since $p(X)=^{*}, X$ is mapped into $F=\bar{p}^{-1}\left({ }^{*}\right)$. From the induced map of the exact sequences of the pairs $(E, X)$ and $(\bar{E}, F)$, we conclude that $F$ has the homotopy type of $X$, all spaces having the homotopy type of CW-complexes.)

To study spaces which admit $A_{n}$-structures, we can work directly with the maps $p_{i}$. In the case of a topological group, this amounts to working only with the classifying bundle and never mentioning group operations. This would be an exercise in rectitude of thought of which it would be pointless to countenance the austerity, for not only would it eliminate a useful perspective on the subject, but, by disguising its own main point, it would place the reader beneath a cloud 
of unknowing. A similar remark can be made about $A_{n}$-spaces. We shall see that an $A_{n}$-structure on a space $X$ is equivalent to an " $A_{n}$-form," that is, a sequence of maps $M_{2}, \cdots, M_{n}$ where each $M_{i}: I^{i-2} \times X^{i} \rightarrow X$ is appropriately defined on $\partial I^{i-2} \times X^{i}$ in terms of $M_{j}$ for $j<i$. (In particular, as indicated above, an $A_{2}$ space is equivalent to an $H$-space, an $A_{3}$-space to a homotopy associative $H$-space $[15 ; 14]$.)

Our study of $A_{n}$-spaces depends strongly on the interplay between $A_{n}$-structures and $A_{n}$-forms. In particular, using both view points we are able to provide an example for each prime $p$ of a space which admits $A_{p-1}$ - but not $A_{p}$-structures. ( $S^{7}$ is a good example for $p=3$.)

The main theorem, Theorem 5, was strongly suggested by the work of Sugawara. The $A_{n}$-forms we will discuss are a greatly simplified version of the appropriate part of his conditions for a group-like space [14]. Where our proofs are suggested by his, we have attempted extensive simplification. The work of Dold and Lashof [3] also had a deep influence on the development of this subject; it is particularly apparent in the proof of Theorem 10. This paper represents in part joint work with J. F. Adams, whose inspiration has permeated this entire effort, though he should not be held responsible for the present exposition. The examples of Theorem 17 are entirely due to him, and his comments were most helpful in the treatment of the complexes $K_{i}$ and construction 8 .

I would also like to express my gratitude to Professor J. C. Moore for suggesting a problem which led to the present paper and for his continuing advice and encouragement while supervising my thesis for Princeton University, from which much of this material is drawn, to Dr. I. M. James for contributing his work on "retractile" subsets [6] at a most opportune time and especially to Dr. S. Y. Husseini, to whom fell the thankless task of reading my preliminary attempts at exposition of this subject.

2. $A_{n}$-forms. Before defining $A_{n}$-forms explicitly, we introduce for each $i \geqq 2$ a special cell complex $K_{i}$ which is homeomorphic to $I^{i-2}$. The reader is on friendly terms with the standard simplices $\Delta^{i}$ and the standard cubes $I^{i}$. He should think of the standard cells $K_{l}$ as similar objects, also having faces and degeneracies and suitable for use as models for a singular homology theory. He should also keep in mind the important differences that

(1) the index $i$ does not refer to the dimension of the cell but rather to the number of factors of $X$ with which $K_{i}$ will be significantly associated later,

(2) $K_{i}$ has $i$ degeneracy operators $s_{1}, \cdots, s_{i}$ defined on it, and

(3) $K_{i}$ has $i(i-1) / 2-1$ faces.

We see already that the complexes $K_{i}$ are more complicated than simplices or cubes. Even to index the faces of $K_{i}$ is not straightforward; the following description of this indexing is the only one we know of which has some intuitive content. Consider a word with $i$ letters, and all meaningful ways of inserting one 
set of parentheses. To each such insertion except for $\left(x_{1} \cdots x_{i}\right)$, there corresponds a cell of $L_{i}$, the boundary of $K_{i}$. If the parentheses enclose $x_{k}$ through $x_{k+s-1}$, we regard this cell as the homeomorphic image of $K_{r} \times K_{s}(r+s=i+1)$ under a map which we call $\partial_{k}(r, s)$. Two such cells intersect only on their boundaries and the "edges" so formed correspond to inserting two sets of parentheses in the word. Thus we have the relations

3(a) $\partial_{j}(r, s+t-1)\left(1 \times \partial_{k}(s, t)\right)=\partial_{j+k-1}(r+s-1, t)\left(\partial_{j}(r, s) \times 1\right)$,

(b) $\partial_{j+s-1}(r+s-1, t)\left(\partial_{k}(r, s) \times 1\right)=\partial_{k}(r+t-1, s)\left(\partial_{j}(r, t) \times 1\right)(1 \times T)$ where $T: K_{s} \times K_{t} \rightarrow K_{t} \times K_{s}$ permutes the factors.

This is enough to obtain $K_{i}$ by induction. Start with $K_{2}$ as a point, *. Given $K_{2}$ through $K_{i-1}$, construct $L_{i}$ by fitting together copies of $K_{r} \times K_{s}$ as indicated by the above conditions. Take $K_{i}$ to be the cone on $L_{i}$.

Proposition 3. $K_{i}$ is homeomorphic to $I^{i-2}$. Degeneracy maps $s_{j}: K_{i} \rightarrow K_{i-1}$ for $1 \leqq j \leqq i$ can be defined so that the following relations hold:

3(c) $s_{j} s_{k}=s_{k} s_{j+1}$ for $k \leqq j$,

(d) $s_{j} \partial_{k}(r, s)=\partial_{k-1}(r-1, s)\left(s_{j} \times 1\right)$ for $j<k$ and $r>2$,

(e) $s_{j} \partial_{k}(r, s)=\partial_{k}(r, s-1)\left(1 \times s_{j-k+1}\right)$ for $s>2, k \leqq j<k+s, s_{j} \partial_{k}(i-1,2)$ $=\pi_{1}$ for $1<j=k<i$ and $1<j=k+1 \leqq i, \quad s_{1} \partial_{2}(2, i-1)=\pi_{2}$ and $s_{i} \partial_{1}(2, i-1)=\pi_{2}$ (where $\pi_{m}$ for $m=1,2$ is projection onto the mth factor), (f) $s_{j} \partial_{k}(r, s)=\partial_{k}(r-1, s)\left(s_{j-s+1} \times 1\right)$ for $k+s \leqq j$.

We will prove Proposition 3 later in this paper by explicitly constructing the complexes $K_{i}$ as subsets of $I^{i-2}$.

REMARK 4. The above relations are reminiscent of the usual ones between the face and degeneracy operators of a semi-simplicial complex. Because the semisimplicial operators correspond, for example, to " $\bar{\partial}_{j}\left(\Delta^{q}\right)$ is the $j$ th face of $\Delta^{q}$ " rather than " $\bar{\partial}_{j}$ imbeds $\Delta^{q-1}$ as the $j$ th face of $\Delta^{q}$," they compose in the opposite direction. With this change, the semi-simplical operators (call them $\bar{\partial}_{j}$ and $\bar{s}_{j}$ ) satisfy $3(\mathrm{a})-(\mathrm{f})$ if we replace $\partial_{j+1}(i-1,2)$ by $\bar{\partial}_{j}$ and $s_{j+1}$ by $\bar{s}_{j}$.

The complexes $K_{i}$ are important because of their role in the following theorem.

THEOREM 5. A space $X$ admits an $A_{n}$-structure if and only if there exist maps $M_{i}: K_{i} \times X^{i} \rightarrow X$ for $2 \leqq i \leqq n$ such that

(1) $M_{2}(*, e, x)=M_{2}(*, x, e)=x$ for $x \in X, *=K_{2}$,

(2) for $\rho \in K_{r}, \sigma \in K_{s}, r+s=i+1$, we have

$$
\begin{aligned}
& M_{i}\left(\partial_{k}(r, s)(\rho, \sigma), x_{1}, \cdots, x_{i}\right)= \\
& M_{r}\left(\rho, x_{1}, \cdots, x_{k-1}, M_{s}\left(\sigma, x_{k}, \cdots, x_{k+s-1}\right), x_{k+s}, \cdots, x_{i}\right),
\end{aligned}
$$

(3) for $\tau \in K_{i}$ and $i>2$, we have

$$
M_{i}\left(\tau, x_{1}, \cdots, x_{j-1}, e, x_{j+1}, \cdots, x_{i}\right)=M_{i-1}\left(s_{j}(\tau), x_{1}, \cdots, x_{j-1}, x_{j+1}, \cdots, x_{i}\right) .
$$


We call such a set of maps an $A_{n}$-form on $X$ and the pair $\left(X,\left\{M_{i}\right\}\right)$ an $A_{n}$-space. [Using conditions 3(a)-(f), the reader may readily check for himself that 5(2)and(3) are consistent; that is, they give a well-defined map of $K_{i} \times X^{[i]} \cup L_{i} \times X^{i}$ into $X$. (Here $X^{[i]}$ is the subset of $X^{i}$ consisting of points with at least one coordinate being e.)]

REMARK 6. Notice that an $A_{2}$-space is just an $H$-space. We will often write $x y$ for $M_{2}(*, x, y)$. Formula $5(2)$ is a bit opaque, but least so when $s=2$ in which case it reduces to

$$
M_{i}\left(\partial_{k}(i-1,2),(\rho, *), x_{1}, \cdots, x_{i}\right)=M_{i-1}\left(\rho, x_{1}, \cdots, x_{k} x_{k+1}, \cdots, x_{i}\right) .
$$

Now for $i=3, K_{3}$ is homeomorphic to $I$ and 5(2) says that $M_{3}: I \times X^{3} \rightarrow X$ is a homotopy between $M_{2}\left(M_{2} \times 1\right)$ and $M_{2}\left(1 \times M_{2}\right)$, that is, between $(x y) z$ and $x(y z)$. Thus $M_{3}$ is an associating homotopy; $M_{2}$ is a homotopy associative multiplication. For the case $i=4$, consider the five ways of associating a product of four factors. If the multiplication is homotopy associative, these five products are related by the following string of homotopies:

$$
x(y(z t)) \cong x((y z) t) \cong(x(y z)) t \cong((x y) z) t \cong(x y)(z t) \cong x(y(z t)) .
$$

Thus we have defined a map of $S^{1} \times X^{4}$ into $X$; the map $M_{4}$ can be regarded as an extension of this map to $I^{2} \times X^{4}$.

Of course any associative $H$-space admits $A_{n}$-forms for any $n$; we need only define $M_{i}\left(\tau, x_{1}, \cdots, x_{i}\right)=x_{1} x_{2} \cdots x_{i}$. We call this a trivial $A_{n}$-form.

Condition 5(3) is technically very useful, but actually is no restriction; that is,

Lemma 7. Suppose $\left\{M_{i}, i<n\right\}$ is an $A_{n-1}$-form and that $M_{n}^{\prime}: K_{n} \times X^{n} \rightarrow X$ satisfies 5(2), then there exists $M_{n}: K_{n} \times X^{n} \rightarrow X$ satisfying 5(2) and (3).

This follows from [6] as we shall indicate in more detail in an Appendix.

3. Derived $A_{n}$-structures. We relate $A_{n}$-forms to $A_{n}$-structures by a specific construction. We are grateful to J. F. Adams for a suggestion which has greatly simplified the construction we originally developed. Milnor defines the $n$-fold join $X * n * X$ by means of certain identifications on $\Delta^{n-1} \times X^{n}$. We will construct $A_{n}$-structures $\mathfrak{p}_{i}: \mathscr{E}_{i} \rightarrow \mathscr{B}_{i}$ in which the space $\mathscr{E}_{i}$ will also have the homotopy type of $X * i * X$ but we will use $K_{i+1} \times X^{i}$ instead of $\Delta^{i-1} \times X^{i}$ and will add further identifications which correspond to the reduced join.

CONSTRUCTION 8. The $A_{n}$-structure derived from an $A_{n}$-form $\left\{M_{i}\right\}$ on $X$.

Let $R=L_{i+1} \times X^{i} \cup K_{i+1} \times X \times X^{[i-1]}$. Define spaces $\mathscr{E}_{i}$ for $1 \leqq i \leqq n$ by means of relative homeomorphisms

$$
\left(K_{i+1} \times X^{i}, R\right) \stackrel{\alpha_{i}}{\longrightarrow}\left(\mathscr{E}_{i}, \mathscr{E}_{i-1}\right)
$$

where $\alpha_{i} \mid R$ is defined by 


$$
\begin{aligned}
& \alpha_{i}\left(\partial_{k}(r, s)(\rho, \sigma), x_{1}, \cdots, x_{i}\right)=\alpha_{r-1}\left(\rho, x_{1}, \cdots, M_{s}\left(\sigma, x_{k}, \cdots, x_{k+s-1}\right), \cdots, x_{i}\right), \\
& \alpha_{i}\left(\tau, x_{1}, \cdots, x_{j-1}, e, x_{j+1}, \cdots, x_{i}\right)=\alpha_{i-1}\left(s_{j}(\tau), x_{1}, \cdots, x_{j-1}, x_{j+1}, \cdots, x_{i}\right), \quad j>1,
\end{aligned}
$$

with the convention that the undefined expression $M_{s}\left(\sigma, x_{k}, \cdots, x_{i+1}\right)$ is to be omitted. [ $\mathscr{E}_{1}$ is just $X$. If we identify $K_{3}$ with $I$, then we can regard $\alpha_{2}$ as identifying $0 \times X^{2}$ with $X$ by the map $(0, x, y) \rightarrow x ; 1 \times X^{2}$ with $X$ by the map $(1, x, y) \rightarrow$ $x y$; and $I \times(X \vee X)$ with $X$ in the obvious way.]

We define spaces $\mathscr{B}_{i}$ for $1 \leqq i \leqq n$ similarly in terms of relative homeomorphisms

$$
\left(K_{i+1} \times X^{i-1}, S\right) \stackrel{\beta}{\longrightarrow}\left(\mathscr{B}_{i}, \mathscr{B}_{i-1}\right)
$$

where $S=L_{i+1} \times X^{i-1} \cup K_{i+1} \times X^{[i-1]}$. The restriction $\beta_{i} \mid S$ is defined by the formulas (9) except that we replace $\alpha$ by $\beta$ throughout and omit $x_{1}$ and all terms $M_{s}\left(\sigma, x_{1}, \cdots, x_{s}\right) .\left[\mathscr{B}_{1}\right.$ is a point and $\mathscr{B}_{2}$ can be recognized as $S X$.] Notice that the definition of $\mathscr{B}_{i}$ uses the maps $M_{j}$ only for $j<i$, hence even if $X$ admits only $A_{n}$-forms, $\mathscr{B}_{n+1}$ can still be constructed, although $\mathscr{E}_{n+1}$ cannot.

Let $\rho_{i}: K_{i+1} \times X^{i} \rightarrow K_{i+1} \times X^{i-1}$ be defined by omitting $x_{1}$, i.e., $\rho_{i}\left(\tau, x_{1}, \cdots, x_{i}\right)$ $=\left(\tau, x_{2}, \cdots, x_{i}\right)$. We see that $\rho_{i}$ induces $\mathfrak{p}_{i}: \mathscr{E}_{i} \rightarrow \mathscr{B}_{i}$. [Notice that $\beta_{i} \mid S$ can be defined by $\beta_{i}\left(\tau, x_{2}, \cdots, x_{i}\right)=\mathfrak{p}_{i-1} \alpha_{i}\left(\tau, e, x_{2}, \cdots, x_{i}\right)$. Hence, by induction, $\mathfrak{p}_{i}$ is well defined. Of course, in the above discussion we have used implicitly the fact that $\alpha_{i} \mid R$ and $\beta_{i} \mid S$ are well-defined maps. This can be proved in much the same way that the reader proved $M_{i} \mid K_{i} \times X^{[i]} \cup L_{i} \times X^{i}$ was well defined.]

In order to show that we have in fact constructed an $A_{n}$-structure on $X$, we need

THEOREM 10. If $X$ is arc-connected, $\mathfrak{p}_{i *}: \pi_{q}\left(\mathscr{E}_{i}, X\right) \rightarrow \pi_{q}\left(\mathscr{B}_{i}\right)$ is an isomorphism for all ${ }_{q}$.

(In fact we will show that $\mathfrak{p}_{i}$ is a quasifibring [4].)

We also must show that $\mathscr{E}_{i-1}$ is contractible in $\mathscr{E}_{i}$; we prove a stronger result.

THEOREM 11. If $X$ is arc-connected, then $\left(\mathscr{E}_{n}, \cdots, \mathscr{E}_{1}\right)$ has the homotopy type of $(X * n * X, \cdots, X)$.

Corresponding to this in the base we have

THEOREM 12. $\left(\mathscr{B}_{i+1}, \mathscr{B}_{i}\right)$ has the homotopy type of $\left(C \mathscr{E}_{i} \cup_{\mathfrak{p}_{i}} \mathscr{B}_{i}, \mathscr{B}_{i}\right)$.

(For any map $f: X \rightarrow Y$, the mapping cone $C X \cup_{f} Y$ is defined as the space obtained from the disjoint union of $C X$ and $Y$ by identifying $X$ as the base of the cone with its image under $f$ in $Y$.)

The base spaces $\mathscr{B}_{i}$ will be very useful invariants associated with an $A_{n}$-space; we give them a special name.

DEFINITION 13. The $X$-projective $i$-space $X P(i), i \leqq n$, associated with an 
$A_{n}$-space is the base space $\mathscr{B}_{i+1}$ of the derived $A_{n}$-structure. $\left(\mathscr{B}_{n+1}\right.$ can be defined even when $\mathfrak{p}_{n+1}$ cannot; it has the homotopy type of $C \mathscr{E}_{n} \cup_{\mathfrak{p}_{n}} \mathscr{B}_{n}$.)

The justification of this terminology arises from considering the classical fibrings of $S^{d-1} * i * S^{d-1}=S^{d i-1}$ by $S^{d-1}$ for $d=1,2,4 . S^{d-1} P(i)$ is respectively real, complex, or quaternionic projective space of $i$-dimensions, and the fibrings can be identified with our construction by using the strictly associative multiplication on $S^{d-1}$.

Convention. If $X$ admits some $A_{n}$-form, we will refer to the $X$-projective $i$-space $X P(i)$ without emphasizing the particular $A_{i}$-form to which $X P(i)$ corresponds.

4. Proof of Theorem 5. Theorems 11 and 12 show that the existence of an $A_{n}$-form implies the existence of an $A_{n}$-structure. To prove the converse, we first observe that it is sufficient to construct an $A_{n}$-form $\left\{M_{i}\right\}$ on some space $F$ of the same homotopy type as $X$, for we can then define an $A_{n}$-form $\left\{N_{i}\right\}$ on $X$ by suitable deformations of the maps

$$
K_{i} \times X^{i} \stackrel{1 \times j^{i}}{\longrightarrow} K_{i} \times F^{i} \stackrel{M_{i}}{\longrightarrow} F \stackrel{s}{\rightarrow} X
$$

where $j: X \rightarrow F$ and $s: F \rightarrow X$ are homotopy inverses. Thus, in light of Proposition 2 , we might as well assume that $X \rightarrow E_{i} \stackrel{p_{i}}{\rightarrow} B_{i}$ is a fibring.

Assume by induction that $M_{j}$ is defined (and $\mathfrak{p}_{j}: \mathscr{E}_{j} \rightarrow \mathscr{B}_{j}$ is constructed) for $j<i$, and that we have commutative diagrams

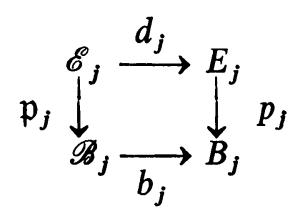

such that $d_{j} \mid \mathscr{E}_{j-1}=d_{j-1}$, etc. The induction begins with the commutative diagram

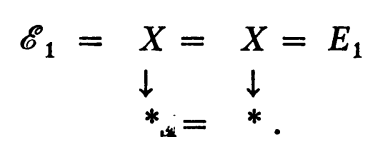

Let $J=$ interior of $\partial_{1}(2, i)\left(K_{2} \times K_{i}\right)$. Note that on all of $R$ except for $J \times\left(X^{i}-X^{[i]}\right), \alpha_{i}$ is defined without using $M_{i}$. There is no difficulty in extending from this subset to $\gamma:\left(L_{i+1}-J\right) \times X^{i} \cup K_{i+1} \times X^{[i]} \rightarrow C \mathscr{E}_{i-1}$. Let $k: C E_{i-1} \rightarrow E_{i}$ be the contraction. Define $j: K_{i+1} \times X^{i-1} \rightarrow B_{i}$ by

$$
j\left(\tau, x_{2}, \cdots, x_{i}\right)=k_{\circ} C d_{i-1} \circ \gamma\left(\tau, e, x_{2}, \cdots, x_{i}\right)
$$

so that $j \circ \rho_{i}=p_{i} \circ k_{\circ} C d_{i-1} \circ \gamma$ over the whole domain of $\gamma$. Thus $j$ induces an extension $b_{i}: \mathscr{B}_{i} \rightarrow B_{i}$ of $b_{i-1}$. Since $L_{i+1}-J$ is a deformation retract of $K_{i+1}$, $k_{\circ} C d_{i-1} \circ \gamma$ can be extended to a map $d: K_{i+1} \times X^{i} \rightarrow E_{i}$ covering $j \circ \rho_{i}$. Thus 
$d\left(\partial_{1}(2, i)\left(K_{2} \times K_{i}\right) \times X^{i}\right) \subset X$ and the desired map $M_{i}$ can be defined by $M_{i}\left(\tau, x_{1}, \cdots, x_{i}\right)=d\left(\partial_{1}(2, i)(*, \tau), x_{1}, \cdots, x_{i}\right)$. The space $\mathscr{E}_{i}$ can now be constructed, and we see that $d$ induces a map $d_{i}: \mathscr{E}_{i} \rightarrow E_{i}$ such that

$$
\mathfrak{p}_{i} \underset{\mathscr{B}_{i}}{\downarrow_{b_{i}}} \underset{B_{i}}{\stackrel{d_{i}}{\rightarrow}} E_{i} p_{i}
$$

is commutative.

Notice that Theorem 5 has the following corollaries (which are already known $[15 ; 14])$.

CoRollary 15. A space $X$ admits a multiplication if and only if there is a map $\mathfrak{p}:(X * X, X) \rightarrow(S X, *)$ such that $\mathfrak{p}_{*}: \pi_{q}(X * X, X) \rightarrow \pi_{q}(S X)$ is an isomorphism for all $q$.

Corollary 16. A space $X$ admits a homotopy associative multiplication if and only if there is a map $\overline{\mathrm{p}}:(X * X * X, X * X, X) \rightarrow\left(C(X * X) \cup_{\mathfrak{p}} S X, S X, *\right)$ such that $\mathfrak{p}=\overline{\mathfrak{p}} \mid X * X$ and $\mathfrak{p}_{*}: \pi_{q}(X * X, X) \rightarrow \pi_{q}(S X)$ is an isomorphism for all $q$ and $\overline{\mathfrak{p}}_{*}: \pi_{q}(X * X * X, X) \rightarrow \pi_{q}\left(C(X * X) \cup_{p} S X\right)$ is an isomorphism for all $q$.

5. Examples. Theorem 5 also shows the relation between the nonexistence of a homotopy associative multiplication on $S^{7}$ and the impossibility of fibring $S^{23}$ by $S^{7}$ over the Cayley projective plane. This suggests that the nonexistence of a homotopy associative multiplication on $S^{7}$ can be shown using Steenrod operations. In fact, this approach can be generalized to indicate more fully the nontrivial distinctions involved in the $A_{n}$-classification.

THEOREM 17. For each prime $p$, there exist spaces which admit $A_{p-1}$-struc- $^{-}$ tures but not $A_{p}$-structures.

Proof. The examples we give are due to J. F. Adams, as are the techniques used in the proof. These spaces are constructed quite explicitly in [1], where further details of the assertions below also can be found. (The above theorem is the part of our joint work referred to there.)

Let $p$ be an odd prime and $Q^{p}$, the group of all those fractions which, in lowest terms, have denominators prime to $p$. Let $X$ be a Moore space with one nonvanishing homology group $Q^{p}$ in dimension $2 n-1$. Since $Q^{p}$ has no $p$-torsion and is infinitely divisible by all other primes, $H_{*}\left(X ; Z_{q}\right)=0$ if $q$ is prime to $p$ and $H_{i}\left(X ; Z_{p}\right)=0$ except for $i=0$ or $2 n-1$, while $H_{2 n-1}\left(X ; Z_{p}\right)=Z_{p}$. [This can be seen by mapping $Q^{p}$ onto $Z_{p}$ by $a / b \rightarrow a b^{\prime}(p)$ where $b b^{\prime} \equiv 1 \bmod p$. $]$ We can use $\mathscr{C}$-theory [12] to deduce that for $i>2 n-1, \pi_{i}(X)$ is isomorphic to the $p$-primary component of $\pi_{i}\left(S^{2 n-1}\right)$. 
Let us imbed $X$ in the space $Z=\Omega^{2} S^{2} X$ in the usual way. Since $Z$ is a loop space, it admits an $A_{n}$-structure for any $n$; call the corresponding maps $N_{i}: K_{i}$ $\times Z^{i} \rightarrow Z$ and let $M_{i}^{\prime}: K_{i} \times X^{i} \rightarrow Z$ denote the restrictions. We would like to deform the maps $M_{i}^{\prime}$ into $X$ so as to obtain $A_{p-1}$-forms on $X$. By induction, assume the image of $M_{j}$ lies in $X$ for $j<i$. Let $T=L_{i} \times X^{i} \cup K_{i} \times X^{[i]} . M_{i}^{\prime}$ is defined on $T$ in terms of $M_{j}$ for $j<1$, so $M_{i}(T) \subset X$. The obstructions to deforming $M_{i}$ into $X$ rel $T$ appear as classes in $H^{q}\left(K_{i} \times X^{i}, T ; \pi_{q}(Z, X)\right)$ which, since $\left(K_{i}, L_{i}\right)$ is isomorphic to $\left(I^{i-2}, I^{i-2}\right)$, is isomorphic to $H^{q+2-i}\left(X^{(i)} ; \pi_{q}(Z, X)\right)$. Since the $p$-primary component of $\pi_{q}\left(\Omega^{2} S^{2 n+1}, S^{2 n-1}\right)=0$, for $q<2 p n-2$ [10], we can conclude that $\pi_{q}(Z, X)=0$ for $q<2 p n-2$. Since $X$ has nontrivial cohomology only in dimension $2 n-1, H^{q}\left(X^{(i)} ; G\right)=0$ for any coefficient group $G$ if $q \neq i(2 n-1)$. Thus the obstructions we have considered lie in trivial groups unless $i(2 n-1)+i-2 \geqq 2 p n-2$, i.e., $i \geqq p$. There are no obstructions to obtaining $A_{p-1}$-forms on $X$.!

Now suppose $X$ did admit an $A_{p}$-form (equivalently, an $A_{p}$-structure). Consider the corresponding maps $\mathfrak{p}_{i}: \mathscr{E}_{i} \rightarrow \mathscr{B}_{i}$. According to Proposition 2, we can replace $\mathfrak{p}_{i}$ by a homotopy equivalent map $\overline{\mathfrak{p}}_{i}$ which is a fibring in the sense of Serre. Therefore in the argument below we can assume without loss of generality that $\mathfrak{p}_{i}$ is a fibring. Thus we know that the Thom-Grysin sequence [2, Exposé 8] applies to the "Thom Space" $C \mathscr{E}_{i} \cup \mathfrak{p}_{i} \mathscr{B}_{i}=X P(i)$. This sequence can be used to compute the cohomology ring of real or complex projective space [9, Theorem 23]. In exactly the same way, since $H^{*}\left(X ; Z_{p}\right) \approx H^{*}\left(S^{2 n-1} ; Z_{p}\right)$, we find that $H^{*}\left(X P(i) ; Z_{p}\right)$ is a truncated polynomial algebra on a generator $u \in H^{2 n}\left(X P(i) ; Z_{p}\right)$ with $u^{i} \neq 0$. But if we choose $n$ prime to $p(p-1)$, the Adem relations on the Steenrod operations $\bmod p$ imply that $u^{p}$ must vanish; therefore $X$ cannot admit $A_{p^{-}}$ structures.

(For $X=S^{7}$, the above argument shows that $S^{7}$ admits no homotopy associative multiplication $[5$, Theorem (1.4)].)

Finally, we remark that if the $A_{n}$-form is trivial, our construction 8 reduces to that of Dold and Lashof.

In that case and more generally whenever $X$ admits a structure $\left\{p_{i} ; i=1,2, \cdots\right\}$ satisfying the usual conditions but for all positive integers $i$, then $X$ has the homotopy type of $\Omega B_{\infty}$ where $B_{\circlearrowleft}$ is the limit of the base spaces $B_{i}[15$, Lemma 10]. We will investigate this relationship more fully in Homotopy associativity of $H$-spaces. II, a sequel to the present paper.

\section{Complexes $K_{i}$.}

Proof of Proposition 3. We exhibit particular representatives of the complexes $K_{i}$ as subsets of $I^{i-2}$. Figure 18 pictures the cases $i=2,3,4,5 ; K_{i}$ being heavily outlined as a subset of $I^{i-2}$.

Let $K_{i}$ be the subset of $I^{i-2}$ consisting of points $\left(t_{1}, \cdots, t_{i-2}\right)$ such that $2^{j} t_{1} \cdots t_{j} \geqq 1$ for $1 \leqq j \leqq i-2 . L_{i}$, the boundary of $K_{i}$, consists of the point of 

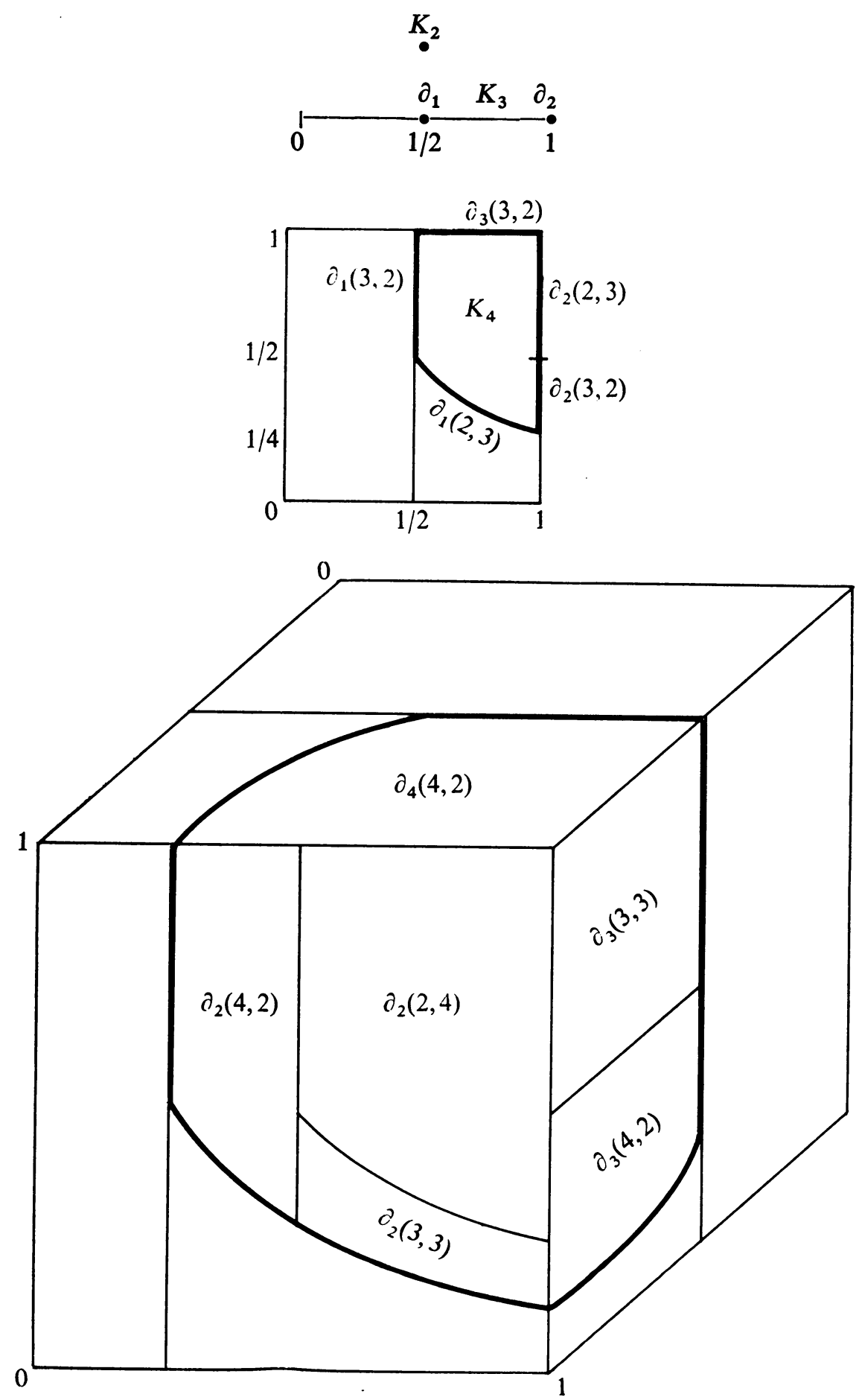

FIGURE 18 
$K_{i}$ such that for some $j$ either $2{ }^{j} t_{1} \cdots t_{j}=1$ or $t_{j}=1$. We give the face operators as follows:

$k=1: \partial_{1}(r, s)\left(K_{r} \times K_{s}\right)$ is to be the subset of $K_{i}$ such that $2^{s-1} t_{1} \cdots t_{s-1}=1$. Let $\rho=\left(t_{1}, \cdots, t_{r-2}\right) \in K_{r}$ and $\sigma=\left(u_{1}, \cdots, u_{s-2}\right) \in K_{s}$ then

$$
\partial_{1}(r, s)(\rho, \sigma)=\left(u_{1}, \cdots, u_{s-2}, u, t_{1}, \cdots, t_{r-2}\right)
$$

with $u=1 / 2^{s-1} u_{1} \cdots u_{s-2}$.

$k>1: \partial_{k}(r, s)\left(K_{r} \times K_{s}\right)$ is the subset of the "face" $t_{k-1}=1$ such that $\left(t_{k}, \cdots, t_{k+s-3}\right) \in K_{s}$ and $\left(t_{1}, \cdots, t_{k-2}, 2^{s-1} t_{k} \cdots t_{k+s-2}, t_{k+s-1}, \cdots, t_{i-2}\right) \in K_{r}$. For $\rho, \sigma$ as above we have

$$
\partial_{k}(r, s)(\rho, \sigma)=\left(t_{1}, \cdots, t_{k-2}, 1, u_{1}, \cdots, u_{s-2}, \bar{t}_{k-1}, t_{k}, \cdots, t_{r-2}\right)
$$

where $\bar{t}_{k-1}=t_{k-1} / 2^{s-1} u_{1} \cdots u_{s-2}$.

Relations 3(a) and (b) can be verified directly from the definitions. For example with $k, j>1$ and

$$
\rho=\left(t_{1}, \cdots, t_{r-2}\right), \quad \sigma=\left(u_{1}, \cdots, u_{s-2}\right), \quad \tau=\left(v_{1}, \cdots, v_{t-2}\right)
$$

we have

$$
\begin{aligned}
\partial_{j}(r, s & +t-1)\left(1 \times \partial_{k}(s, t)\right)(\rho, \sigma, \tau) \\
& =\left(t_{1}, \cdots, t_{j-2}, 1, u_{1}, \cdots, u_{k-2}, 1, v_{1}, \cdots, v_{t-2}, \bar{u}_{k-1}, u_{k}, \cdots, u_{s-2}, \bar{t}_{j-1}, t_{j}, \cdots, t_{r-2}\right)
\end{aligned}
$$

with

$$
\bar{u}_{k-1}=u_{k-1} / 2^{t-1} v_{1} \cdots v_{t-2} \text { and } \bar{t}_{j-1}=t_{j-1} / 2^{s-1} u_{1} \cdots u_{s-2} \text {. }
$$

On the other hand

$$
\begin{aligned}
\partial_{j+k-1} & (r+s-1, t)\left(\partial_{j}(r, s) \times 1\right)(\rho, \sigma, \tau) \\
& =\left(t_{1}, \cdots, t_{j-2}, 1, u_{1}, \cdots, u_{k-2}, 1, v_{1}, \cdots, v_{t-2}, \bar{u}_{k-1}, u_{k}, \cdots, u_{s-2}, \bar{t}_{j-1}, t_{j}, \cdots, t_{r-2}\right)
\end{aligned}
$$

where

$$
\overline{\bar{t}}_{j-1}=t_{j-1} / 2^{s+t-2} u_{1} \cdots u_{k-2} v_{1} \cdots v_{t-2} \bar{u}_{k-1} u_{k} \cdots u_{s-2} .
$$

Expanding $\bar{u}_{k-1}$ we see that relation 3(a) holds.

The degeneracy maps $s_{j}: K_{i+1} \rightarrow K_{i}$ can be defined on $L_{i+1}$ using 3(d)-(f), since the latter are compatible with $3(\mathrm{a})$ and (b). It also follows easily that 3(c) will be satisfied on $L_{i+1}$. The map $s_{j}$ on all of $K_{i+1}$ can now be obtained "by taking the cone." That is, represent $K_{i+1}$ as pairs $(t, \tau)$ with $\tau \in L_{i+1}$ and similarly for $K_{i}$. If $s_{j}(\tau)=\left(s, \tau^{\prime}\right), \tau^{\prime} \in L_{i}$ then define $s_{j}(t, \tau)$ as $\left(t s, \tau^{\prime}\right)$. With this definition it is easy to verify 3 (c) on all of $K_{i+1}$, while $s_{j}$ was constructed so as to satisfy $3(d)-(f)$. The relations are important in that they make the conditions of Theorem 5 consistent. 
7. Proof of Theorem 10. This will follow from:

THEOREM 19. $\mathfrak{p}_{i}: \mathscr{E}_{i} \rightarrow \mathscr{B}_{i}$ is a quasifibring (with fibre $X$ ).

Proof. (Cf. [3].) For $i=1, \mathfrak{p}_{1}$ is trivially a quasifibring since $\mathscr{B}_{1}$ is a point. By induction assume $\mathfrak{p}_{i-1}$ is a quasifibring. We will decompose $\mathfrak{p}_{i}$ as the union of two sub-quasifibrings.

Let $U=\mathscr{B}_{i}-\mathscr{B}_{i-1}$ and $P=\mathfrak{p}_{i}^{-1}(U)=\mathscr{E}_{i}-\mathscr{E}_{i-1}$.

LEMMA 20. $\mathfrak{p}_{i} \mid P$ is a quasifibring.

Proof. Since $\alpha_{i}^{-1}$ is a well-defined homeomorphism on $P$ and $\beta_{i}^{-1}$ is a welldefined homeomorphism on $U$, we see that $\mathfrak{p}_{i} \mid P$ is equivalent to the map $X \times U \rightarrow U$ which is projection onto the second factor, trivially a quasifibring.

Now recall that $R$ denotes the set $L_{i+1} \times X^{i} \cup K_{i+1} \times X \times X^{[i-1]}$ and $S$ denotes the set $L_{i+1} \times X^{i-1} \cup K_{i+1} \times X^{[i-1]}$.

LEMMA 21. There exists a neighborhood $\mathscr{R}$ in $K_{i+1} \times X^{i}$ of $R$ of which $R$ is a strong deformation retract.

Proof. Take a "tubular" neighborhood $N^{\prime}$ of $L_{i+1}$ in $K_{i+1}$, i.e., there is a homeomorphism of $N^{\prime}$ onto $L_{i+1} \times(0,1]$ which maps $L_{i+1}$ identically onto $L_{i+1} \times 1$. Let $N$ be the inverse image of $L_{i+1} \times(1 / 2,1]$. There exists a deformation $h_{s}: K_{i+1} \rightarrow K_{i+1}$ rel $L_{i+1}$ such that $h_{1}$ is the identity, $h_{0}(N) \subset L_{i+1}$ and $h_{s}$ is constant outside $N^{\prime}$.

Since $X$ has the homotopy type of a CW-complex, there exists an open neighborhood $N_{e}$ of $e$ and a deformation $k_{s}: X \rightarrow X$ such that $k_{1}$ is the identity, $k_{0}\left(N_{e}\right)$ $=e[16,(\mathrm{M})$, p. 230]; also cf. [7, Theorem (8.3)].

Take $\mathscr{R}$ as the union of $N \times X^{i}$ and all sets of the form $K_{i+1} \times X \times X \times \cdots$ $\times N_{e} \times \cdots \times X$ except $K_{i+1} \times N_{e} \times X \times \cdots \times X$. Define $D_{t}^{\prime \prime}: K_{i+1} \times X^{i} \rightarrow K_{i+1}$ $\times X^{i}$ by $h_{t} \times 1 \times\left(k_{t}\right)^{i-1}$. We have $D_{1}^{\prime \prime}$ as the identity, $D_{0}^{\prime \prime}(\mathscr{R}) \subset R$ and $D_{t}^{\prime \prime}(R) \subset R$. Therefore $D_{t}^{\prime \prime}$ can be deformed to give a strong deformation retraction $D_{t}^{\prime}$ of $\mathscr{R}$ onto $R$.

Now let $\mathscr{S}$ be obtained from $\mathscr{R}$ by omitting the first $X$ factor, so that $\mathscr{S}$ is an open neighborhood of $S$. A strong deformation retraction $d_{t}^{\prime}$ of $\mathscr{S}$ onto $S$ is given by $d_{t}^{\prime}\left(\tau, x_{2}, \cdots, x_{i}\right)=\mathfrak{p}_{i} D_{t}^{\prime}\left(\tau, e, x_{2}, \cdots, x_{i}\right)$.

Let $Q=\alpha_{i}(\mathscr{R})$ and $V=\beta_{i}(\mathscr{S})$, so that $\mathscr{E}_{i}$ is covered by $P \cup Q$ and $\mathscr{E}_{i-1} \subset Q$ while $\mathscr{B}_{i}$ is covered by $U \cup V$ and $\mathscr{B}_{i-1} \subset V$. Notice that $Q=\mathfrak{p}_{i}^{-1}(V)$. We will prove

LEMMA 22. $\mathfrak{p}_{i} \mid Q$ is a quasifibring.

From this and the fact that $\mathfrak{p}_{i}$ is a quasifibring over $U$ and $U \cap V$, it will follow that $\mathfrak{p}_{i}$ is a quasifibring $[4,(2.2)]$.

To prove Lemma 22 we will need the following criterion: 
LEMMA $23[4,(2.10)] . p: E \rightarrow B$ is a quasifibring if for some $B^{\prime} \subset B$ and $E^{\prime}=p^{-1}\left(B^{\prime}\right), p \mid E^{\prime}$ is a quasifibring and there exist deformations

$$
D_{t}: E \rightarrow E, d_{t}: B \rightarrow B
$$

with

and

$$
\begin{aligned}
& D_{1}=1, \quad D_{t}\left(E^{\prime}\right) \subset E^{\prime}, \quad D_{0}(E) \subset E^{\prime}, \\
& d_{1}=1, \quad d_{t}\left(B^{\prime}\right) \subset B^{\prime}, \quad d_{0}(B) \subset B^{\prime}, \quad p D_{1}=d_{1} p
\end{aligned}
$$

$$
D_{0 *}: \pi_{i}\left(p^{-1}(x)\right) \approx \pi_{i}\left(p^{-1}\left(d_{0}(x)\right)\right) \quad \text { for all } x \in B, i \geqq 0 .
$$

Proof of Lemma 22. We will deform $Q$ onto $\mathscr{E}_{i-1}=\alpha_{i}(R)$. Define $D_{t}: Q \rightarrow Q$ by $D_{t} \circ \alpha_{i}=\alpha_{i} D_{t}^{\prime}$ on $Q-\mathscr{E}_{i-1}$ and as the identity on $\mathscr{E}_{i-1}$. Similarly define $d_{t}: V \rightarrow V$ by $d_{t} \circ \beta_{i}=\beta_{i} d_{t}^{\prime}$ on $V-\mathscr{B}_{i-1}$ and as the identity on $\mathscr{B}_{i-1}$. Both $D_{t}$ and $d_{t}$ are well defined since $D_{t}^{\prime}$ and $d_{t}^{\prime}$ are strong deformation retractions, i.e., constant on $R$ and $S$ respectively. Clearly $\mathfrak{p}_{i} D_{t}=d_{i} \mathfrak{p}_{i}$. We have $D_{1}=1$, $D_{t} \mid \mathscr{E}_{i-1}=1, D_{0}(Q) \subset \mathscr{E}_{i-1}$ and $d_{1}=1, d_{t} \mid \mathscr{B}_{i-1}=1, d_{0}(V) \subset \mathscr{B}_{i-1}$.

Finally, we must consider $g_{z}=D_{0} \mid \mathfrak{p}_{i}^{-1}(z): \mathfrak{p}_{i}^{-1}(z) \rightarrow \mathfrak{p}_{i}^{-1}\left(d_{0}(z)\right)$ for any $z \in V$. If $z \in \mathscr{B}_{i-1}, d_{0}(z)=z$ and $g_{z}$ is the identity, trivially a homotopy equivalence. If $z \in V-\mathscr{B}_{i-1}$, then $z$ can be written uniquely as $\beta_{i}\left(\tau, x_{2}, \cdots, x_{i}\right)$ and for $d_{0}(z)$, there exists an $r$ such that $d_{0}(z)$ can be written uniquely as $\beta_{r}\left(\mu, y_{2}, \cdots, y_{r}\right)$. Define a homeomorphism $f_{z}: X \rightarrow \mathfrak{p}_{i}^{-1}(z)$ by $f_{z}(x)=\alpha_{i}\left(\tau, x, x_{2}, \cdots, x_{i}\right)$ and $h_{z}: X \rightarrow$ $\mathfrak{p}_{i}^{-1}\left(d_{0}(z)\right)$ by $h_{z}(x)=\alpha_{r}\left(\mu, x, y_{2}, \cdots, y_{r}\right)$.

Now recall the definition of $d_{t}^{\prime}$. We see that $d_{0}$ is homotopic rel $\mathscr{B}_{i-1}$ to $d_{0} \beta_{i}\left(\tau, x_{2}, \cdots, x_{i}\right) \rightarrow \beta_{i}\left(h_{0}(\tau), k_{0}\left(x_{2}\right), \cdots, k_{0}\left(x_{i}\right)\right)$. We are concerned with $\left(\tau, x_{2}, \cdots, x_{i}\right)$ if $h_{0}(\tau) \in L_{i+1}$ or if $k_{0}\left(x_{j}\right)=e$. Thus $d_{0}(z)$ can be represented as $\beta_{r}\left(\rho, y_{2}, \cdots, y_{r}\right)$ where each $y_{j}$ is some $x_{k}$ except that at most one $y_{j}$ may have the form $M_{s}\left(\sigma, x_{k+1}, \cdots, x_{k+s}\right)$. Now by means of $f_{z}$ and $h_{z}, g_{z}$ can be identified with a map of $X$ into $X$ given either by $x \rightarrow x$ (trivially a homotopy equivalence) or by $x \rightarrow$ $M_{s}\left(\sigma, x, x_{k+1}, \cdots, x_{k+s}\right)$ for some fixed $\sigma, x_{k+1}, \cdots, x_{k+s}$. The latter is homotopic to $x \rightarrow x\left(x_{k+1}\left(\cdots\left(x_{k+s}\right) \cdots\right)\right)$, again a homotopy equivalence since $X$ is arc-connected (right translation is a homotopy equivalence). Since $\mathfrak{p}_{i-1}=\mathfrak{p}_{i} \mid \mathscr{E}_{i-1}$ is a quasifibring by the inductive assumption, by Lemma 23 we conclude that $p_{i} \mid Q$ is a quasifibring. This completes the proof of Theorem 10 .

8. The homotopy type of $\mathscr{E}_{i}$ and $\mathscr{B}_{i}$. Consider the space, call it $\mathscr{D}_{i}$, defined by a relative homeomorphism

$$
\left(K_{i+1} \times X^{i-1}, S\right) \stackrel{\gamma_{i}}{\rightarrow}\left(\mathscr{D}_{i}, \mathscr{E}_{i-1}\right),
$$

where $\gamma_{i}\left(\tau, x_{2}, \cdots, x_{i}\right)=\alpha_{i}\left(\tau, e, x_{2}, \cdots, x_{i}\right)$.

Proposition 24. $\mathscr{D}_{i}, \mathscr{E}_{i-1}$ has the homotopy type of $C \mathscr{E}_{i-1}, \mathscr{E}_{i-1}$.

In order to prove this, we need an auxiliary map. 
Proposition 25. There exist homeomorphisms $\eta_{i}: I \times K_{i} \rightarrow K_{i+1}$ such that

(1) $\eta_{i}(0, \tau)=\partial_{2}(2, i)(*, \tau)$, and

(2) $\eta_{i}\left(t, \partial_{k}(r, s)(\rho, \sigma)\right)=\partial_{k+1}(r+1, s)\left(\eta_{r}(t, \rho), \sigma\right)$.

Proof. Assume the proposition true for $j<i$. It is easy to verify that equation (2) yields a homeomorphism $\eta_{i}^{\prime}$ of $I \times L_{i}$ into $K_{i+1}$. The image $J_{i}$ consists of all faces $\partial_{k}(r, s)\left(K_{r} \times K_{s}\right)$ with $k>1, r>1$. We can regard $K_{i+1}$ as formed from $I \times J_{i}$ by identifying $\left(0, \eta_{i}^{\prime}(t, \tau)\right)$ with $\left(0, \eta_{i}^{\prime}\left(t, \tau^{\prime}\right)\right)$ for any $\tau, \tau^{\prime} \in L_{i}$. In this way, the face $\partial_{2}(2, i)\left(K_{2} \times K_{i}\right)$ is identified with $C L_{i}$ which in turn we regard as $K_{i}$. Looking at things this way, we obtain $\eta_{i}$ satisfying (1) and (2) by setting $\eta_{i}(t,(s, \tau))=\left(s, \eta_{i}^{\prime}(t, \tau)\right)$ for $\tau \in L_{i},(s, \tau) \in C L_{i}=K_{i}$.

CoROLlaRY 26. $s_{j+1} \eta_{i}(t, \tau)=\eta_{i-1}\left(t, s_{j}(\tau)\right)$.

The verification is straightforward.

Proof of Proposition 24. Define $\phi_{i}: C \mathscr{E}_{i-1} \rightarrow \mathscr{D}_{i}$ by $\phi_{i}\left(t, \alpha_{i-1}(\tau, \chi)\right)=\gamma_{i}\left(\eta_{i}(t, \tau), \chi\right)$ where $\chi=\left(x_{2}, \cdots, x_{i}\right)$. To obtain an inverse for $\phi_{i}$ define $\bar{\psi}_{i}: K_{i+1} \times X^{i-1}$ $\rightarrow C \mathscr{E}_{i-1}$ by $\bar{\psi}_{i}(\tau, \chi)=\left(t, \alpha_{i-1}\left(\tau^{\prime}, \chi\right)\right)$ where $\eta_{i}\left(t, \tau^{\prime}\right)=\tau$. Now part of $\Psi_{i} \mid S$ lies in $C \mathscr{E}_{i-2} \subset C \mathscr{E}_{i-1}$, instead of in $\mathscr{E}_{i-1}$ as it should to induce a map of $\mathscr{D}_{i}$ into $C \mathscr{E}_{i-1}$. However $C \mathscr{E}_{i-2} \subset C \mathscr{E}_{i-1}$ is homotopic to $\phi_{i-1}\left(C \mathscr{E}_{i-2}\right) \subset \mathscr{D}_{i-1} \subset \mathscr{E}_{i-1}$ by the following argument: It is straightforward to verify that $\phi_{i} \mid \mathscr{E}_{i-1}$ is homotopic in $\mathscr{E}_{i-1}$ to the identity. It involves showing, by an inductive argument, that $s_{1}\left(\eta_{i}(1, \tau)\right): K_{i}, L_{i} \rightarrow K_{i}, L_{i}$ is homotopic to the identity in a way which is compatible with $\gamma_{i}$. We conclude that $C\left(\phi_{i-1} \mid \mathscr{E}_{i-2}\right): C \mathscr{E}_{i-2}, \mathscr{E}_{i-2} \rightarrow C \mathscr{E}_{i-2}, \mathscr{E}_{i-2}$ is homotopic to the identity. Next $C\left(\phi_{i-1} \mid \mathscr{E}_{i-2}\right)$ is homotopic in $C \mathscr{E}_{i-1}$ to $\phi_{i}$ by:

LEMMA 27. Let $f: Y \rightarrow Z$ extend to $F: C Y \rightarrow Z$ then $C f: C Y \rightarrow C Z$ is homotopic rel $Y$ to $F$.

Proof. Define a homotopy $F_{r}: C Y \rightarrow C Z$ for $0 \leqq r \leqq 2$ by

$$
\begin{array}{ll}
F_{r}(t, F(r t+1-r, y)), & 0 \leqq r \leqq 1, \\
F_{r+1}(t(1-r)+r, F(t, y)), & 0 \leqq r \leqq 1 .
\end{array}
$$

Thus $\bar{\psi}_{i}$ can be deformed to a map $\psi_{i}: K_{i+1} \times X^{i-1} \rightarrow C \mathscr{E}_{i-1}$ such that $\psi_{i} \mid S$ $=\gamma_{i} \mid S$. It is clear that $\psi_{i}: \mathscr{D}_{i}, \mathscr{E}_{i-1} \rightarrow C \mathscr{E}_{i-1}, \mathscr{E}_{i-1}$, the map induced by $\psi_{i}$, is a homotopy inverse for $\phi_{i}$ as a map of pairs.

Proof of Theorem 12. Recall the definition of $\beta_{i}:\left(K_{i+1} \times X^{i-1}, S\right) \rightarrow\left(\mathscr{B}_{i}, \mathscr{B}_{i-1}\right)$. We see directly that $\beta_{i}$ can be factored as $\sigma_{i} \circ \gamma_{i}$ where $\sigma_{i}:\left(\mathscr{D}_{i}, \mathscr{E}_{i-1}\right) \rightarrow\left(\mathscr{B}_{i}, \mathscr{B}_{i-1}\right)$ is also a relative homeomorphism and $\sigma_{i} \mid \mathscr{E}_{i-1}=\mathfrak{p}_{i-1}$. Since $\left(\mathscr{D}_{i}, \mathscr{E}_{i-1}\right)$ has the homotopy type of $\left(C \mathscr{E}_{i-1}, \mathscr{E}_{i-1}\right)$, this shows that $\left(\mathscr{B}_{i}, \mathscr{B}_{i-1}\right)$ has the homotopy type of $\left(C \mathscr{E}_{i-1} \cup_{p_{i-1}} \mathscr{B}_{i-1}, \mathscr{B}_{i-1}\right)$.

Proof of Theorem 11. We can map $X \times \mathscr{D}_{i}$ into $\mathscr{E}_{i}$ in the obvious way (cor- 
responding to $X \times K_{i+1} \times X^{i-1} \rightarrow K_{i+1} \times X \times X^{i-1}$ ). We can regard $\mathscr{E}_{i}$ as obtained from $X \times \mathscr{D}_{i}$ by identifying

with

$$
\left(x, \gamma_{i}\left(\partial_{1}(r, s)(\rho, \sigma), x_{2}, \cdots, x_{i}\right)\right)
$$

$$
\alpha_{r-1}\left(\rho, M_{s}\left(\sigma, x, x_{2}, \cdots, x_{s}\right), x_{s+1}, \cdots, x_{i}\right) .
$$

The subset on which these identifications are carried out is just $X \times \phi_{i}\left(\mathscr{E}_{i-1}\right)$. Thus $\left(\mathscr{E}_{i}, \mathscr{E}_{i-1}\right)$ has the homotopy type of $X \times C \mathscr{E}_{i-1} \cup_{\mu} \mathscr{E}_{i-1}$ where $\mu: X \times \mathscr{E}_{i-1}$ $\rightarrow \mathscr{E}_{i-1}$ is obtained by

$$
X \times \mathscr{E}_{i-1} \stackrel{1 \times \phi_{i} \mid}{\longrightarrow} X \times \mathscr{D}_{i} \rightarrow \mathscr{E}_{i}
$$

Note that $\mu \mid e \times \mathscr{E}_{i-1}$ is just $\phi_{i} \mid \mathscr{E}_{i-1}$ and hence homotopic to the identity.

To prove that $X \times C \mathscr{E}_{i-1} \cup_{\mu} \mathscr{E}_{i-1}, \mathscr{E}_{i-1}$ has the homotopy type of $X * \mathscr{E}_{i-1}, \mathscr{E}_{i-1}$, we use the Meyer-Vietoris sequence in the obvious way [13, Lemma 3.4], [ $X$ is arc-connected and hence $X \times C \mathscr{E}_{i-1} \cup_{\mu} \mathscr{E}_{i-1}$ and $X * \mathscr{E}_{i-1}$ are simply connected, so that a homology argument is sufficient.] Thus it is sufficient to show

LEMMA 28. The map of $X \times \mathscr{E}_{i-1}$ into $X \times \mathscr{E}_{i-1}$ given by $q(x, z)=(x, \mu(x, z))$ is a homotopy equivalence.

When $i=2$, this is a familiar fact about $H$-spaces. We mimic the proof used in that special case, cf. [15, Lemma 6].

Proof. By checking the formulas, we find that $\mu$ applied to $X \times X \subset X \times \mathscr{E}_{i-1}$ goes into $X$ and is in fact just $M_{2}$, the multiplication on $X$. The induced map $\mu_{*}: \pi_{n}(X)+\pi_{n}\left(\mathscr{E}_{i-1}\right)=\pi_{n}\left(X \times \mathscr{E}_{i-1}\right) \rightarrow \pi_{n}\left(\mathscr{E}_{i-1}\right)$ can be seen by the usual arguments for $H$-spaces to coincide with the usual addition in the homotopy groups, mapping $\pi_{n}(X)$ into a subgroup of $\pi_{n}\left(\mathscr{E}_{i-1}\right)$ in the obvious way. Thus $q_{*}: \pi_{n}(X)$ $+\pi_{n}\left(\mathscr{E}_{i-1}\right) \rightarrow \pi_{n}(X)+\pi_{n}\left(\mathscr{E}_{i-1}\right)$ can be seen to be given by $q_{*}(\alpha, \beta)=(\alpha, \alpha+\beta)$ which is obviously an isomorphism for all $n$. Since in our category all spaces have the homotopy type of a CW-complex, $q$ is a homotopy equivalence.

9. Reduction to the Dold-Lashof construction. We now make more precise our remark that construction 8 reduces to that given by Dold and Lashof [3] if the $A_{n}$-form is trivial. (It is necessary not only that $M_{2}$ be strictly associative, but also that $M_{i}$ be given by $M_{i}\left(\tau, x_{1}, \cdots, x_{i}\right)=x_{1} \cdots x_{i}$.

Recall that Dold and Lashof defined quasifibrings $\bar{p}_{i}: \bar{E}_{i} \rightarrow \bar{B}_{i}$ by the following inductive procedure. Let $M: X \times X \rightarrow X$ be an associative multiplication. Let $\bar{E}_{1}=X, \bar{B}_{1}=*$. Assume by induction that $\bar{p}_{i}: \bar{E}_{i} \rightarrow \bar{B}_{i}$ is defined as well as an associative action $\bar{M}: X \times \bar{E}_{i} \rightarrow \bar{E}_{i}$. Using the unreduced cone, define $\bar{E}_{i+1}$ as $X \times C \bar{E}_{i} \cup \bar{M}_{\bar{M}} \bar{E}_{i}$ and $\bar{B}_{i+1}$ as $C \bar{E}_{i} \cup \bar{p}_{i} \bar{B}_{i}$. Define $\bar{p}_{i+1}$ by $\bar{p}_{i+1}(x, t, z)=(t, z)$ for $z \in \bar{E}_{i}, \quad \bar{p}_{i+1} \mid \bar{E}_{i}=\bar{p}_{i}$ and $\bar{M}^{\prime}: X \times \bar{E}_{i+1} \rightarrow \bar{E}_{i+1}$ by $\bar{M}^{\prime}(x,(y, t, z))=(x y, t, z)$ $x, y \in X, z \in \bar{E}_{i}$. It is easy to verify that the inductive hypotheses are satisfied. 
Dold and Lashof sho that $\bar{p}_{i+1}$ is a quasifibring; they have constructed an $A_{n}$-structure on $X$ for arbitrary $n$.

We have seen in the last section that $\mathscr{E}_{i}$ can be regarded as $X \times C \mathscr{E}_{i-1} \cup_{\mu} \mathscr{E}_{i-1}$. The use of the reduced versus the unreduced cone is irrelevant to the question of homotopy type; let us assume the Dold-Lashof construction reworked using the reduced cone.

By reversing the process used to show that $\mathscr{E}_{i}$ has the homotopy type of $X \times C \mathscr{E}_{i-1} \cup{ }_{\mu} \mathscr{E}_{i-1}$, we can show that, up to homotopy type, $\bar{E}_{i}$ can be defined in terms of a relative homeomorphism

$$
\left(\Delta^{i-1} \times X^{i}, \bar{R}\right) \stackrel{a_{i}}{\rightarrow}\left(\bar{E}_{i}, \bar{E}_{i-1}\right)
$$

where $\bar{R}=\partial \Delta^{i-1} \times X^{i} \cup \Delta^{i-1} \times X \times X^{[i-1]}$ and $a_{i} \mid \bar{R}$ is given as follows: Let $\tau=\left(t_{1}, \cdots, t_{i}\right) \in \Delta^{i-1}, \chi=\left(x_{1}, \cdots, x_{i}\right) \in X^{i}$.

$$
\begin{aligned}
a_{i}(\tau, \chi) & =a_{i-1}\left(t_{1}, \cdots, \hat{t}_{j}, \cdots, t_{1}, x_{1}, \cdots, x_{j} x_{j+1}, \cdots, x_{i}\right), \quad \text { if } t_{j}=0, j<i, \\
a_{i}\left(t_{1}, \cdots, t_{i-1}, 0, \chi\right) & =a_{i-1}\left(t_{1}, \cdots, t_{i-1}, x_{1}, \cdots, x_{i-1}\right), \\
a_{i}(\tau, \chi) & =a_{i-1}\left(t_{1}, \cdots, t_{j-1}+t_{j}, \cdots, t_{i}, x_{1}, \cdots, \hat{x}_{j}, \cdots, x_{i}\right), \text { if } x_{j}=e \text { for } j>1 .
\end{aligned}
$$

Thus to map $\mathscr{E}_{i}$ into $\bar{E}_{i}$, we need only a suitably defined homeomorphism $\mathscr{T}_{i+1}: K_{i+1} \rightarrow \Delta^{i-1}, \mathscr{T}_{2}$ is canonical (!). We take $\mathscr{T}_{3}$ to be the linear map determined by sending $1 / 2$ to $(0,1)$ and 1 to $(1,0)$. Define $\overline{\mathscr{T}}_{i}: L_{i} \rightarrow \partial \Delta^{i-2}$ as follows: Let $\mathscr{T}_{r}(\rho)=\left(t_{1}, \cdots, t_{r-1}\right) \in \Delta^{r-2}$ for $\rho \in K_{r}$ then

$$
\overline{\mathscr{T}}_{i} \partial_{k}(\rho, \sigma)=\left(t_{1}, \cdots, t_{k-1}, 0, \cdots, 0, t_{k}, \cdots, t_{r-1}\right)
$$

[ $s-2$ coordinates are set equal to zero]. This gives a well-defined map of $L_{i}$ onto $\Delta^{i-2}$. It can be described as collapsing each face which is homeomorphic to $K_{r} \times K_{s}$ onto the image of one of its axes $K_{r}$. Hence it is possible to extend to a relative homeomorphism $\mathscr{T}_{i}: K_{i}, L_{i} \rightarrow \Delta^{i-2}, \partial \Delta^{i-2}$.

Now map $\mathscr{E}_{i}$ into $\bar{E}_{i}$ by $\alpha_{i}\left(\tau, x_{1}, \cdots, x_{i}\right) \rightarrow a_{i}\left(\mathscr{T}_{i+1}(\tau), x_{1}, \cdots, x_{i}\right)$. Although $\mathscr{T}_{i+1}^{-1}$ is not uniquely defined, it is easy to construct an inverse to the above map, using the fact that the $A_{n}$-form is trivial, and hence $\alpha_{i}\left(\partial_{k}(r, s)(\rho, \sigma), x_{1}, \cdots, x_{i}\right)$ is independent of $\sigma$.

A similar analysis shows that $\bar{B}_{i}$ has the homotopy type of $\mathscr{B}_{i}$ and that $\bar{p}_{i}$ is equivalent to $\mathfrak{p}_{i}$.

\section{APPENDIX}

Retractile subspaces. In [6], I. M. James has studied a property of pairs of complexes for which we find many uses. We rework his Lemma 3.2 as a definition.

DEFINITION A.1. A subcomplex $L$ is retractile in a complex $K$ if given any null-homotopic map $f: K \rightarrow X$ such that $f \mid L$ is constant then $f$ is null-homotopic rel $L$. 
Proposition A.2 [6, Corollary 4.4]. Let $(X, m)$ be an $H$-space. If $L$ is retractile in $K$, and we are given homotopic maps $f_{0}, f_{1}: K \rightarrow X$ which agree on $L$, then $f_{0} \simeq f_{1}$ rel $L$.

We are more interested in nontrivial homotopies on $L$.

Proposition A.3. L is retractile in $K$ if and only if given any null-homotopic map $f: K \rightarrow X$ and a null-homotopy $g_{t}: L \rightarrow X$ such that $g_{1}=f \mid L$ then $g_{t}$ extends to a null-homotopy $f_{t}: K \rightarrow X$ such that $f_{1}=f$.

Proof. By the homotopy extension theorem, $g_{t}$ extends to $f_{t}^{\prime}: K \rightarrow X$ such that $f_{1}^{\prime}=f$. Since $f_{0}^{\prime}$ is null-homotopic and constant on $L, f_{0}^{\prime}$ is null-homotopic rel $L$. Thus there is a null-homotopy $\bar{f}_{t}: K \rightarrow X$ with $0 \leqq t \leqq 2$ which restricted to $L$ is $g_{t}$ for $0 \leqq t \leqq 1$ and constant for $1 \leqq t \leqq 2$. It is easy to alter $\bar{f}_{t}$ to obtain $f_{t}$ as desired again using the homotopy extension theorem.

Proposition A.4. Let $(X, m)$ be an $H$-space. If $L$ is retractile in $K$ then given homotopic maps $f_{0}, f_{1}: K \rightarrow X$ and a homotopy $g_{t}: L \rightarrow X$ such that $g_{i}=f_{i} \mid L$ for $i=0,1$ then $g_{t}$ extends to a homotopy $f_{t}: K \rightarrow X$.

This is proved by reducing to the previous case, just as James did in proving his Corollary 4.4. [It is necessary to note that his Lemma 3.4 can be generalized to

Lemma A.5. Let $p: X \rightarrow Y$ induce isomorphisms of all homotopy groups. Let $h_{0}, h_{1}: K \rightarrow X$ and $g_{t}: L \rightarrow X$ such that $g_{i}=h_{i} \mid L$ for $i=0,1$. Suppose there exists $j_{t}: K \rightarrow Y$ such that $j_{i}=p h_{i}$ for $i=0,1$ and $j_{t} \mid L=p g_{t}$. Then $g_{t}$ extends to $h_{t}: K \rightarrow X$.]

Proof of Lemma 7. Corresponding to $M_{n}^{\prime} \mid L_{n} \times X^{n} \rightarrow X$ we have a map of $X^{n}$ into $X^{L_{n}}$. The extension to $K_{n} \times X^{n}$ corresponds to a homotopy between this map $f_{0}$ and the one given by $f_{1}\left(x_{1}, \cdots, x_{n}\right)(\tau)=x_{1}\left(x_{2}\left(\cdots\left(x_{n-1} x_{n}\right)\right)\right)$. Since $X^{L_{n}}$ is an $H$-space (because $X$ is) and $X^{[i]}$ is retractile in $X^{i}[6$, Lemma 3.1], Proposition A. 4 says that there is a homotopy between $f_{0}$ and $f_{1}$ which corresponds to a map $M_{n}: K_{n} \times X^{n} \rightarrow X$ satisfying $5(2)$ and (3) as desired.

\section{BIBLIOGRA PHY}

1. J. F. Adams, The sphere, considered as an H-space mod p, Quart. J. Math. Oxford Ser. (2) 12 (1961), 52-60.

2. H. Cartan, Séminaire, École Normale Supérieure 1959-60.

3. A. Dold and R. Lashof, Principal quasifibrations and fibre homotopy equivalence of bundles, Illinois J. Math. 3 (1959), 285-305.

4. A. Dold and R. Thom, Quasifaserungen und unendliche symmetrische Produkte, Ann. of Math. (2) 67 (1958), 239-281.

5. I. M. James, Multiplications on spheres. II, Trans. Amer. Math. Soc. 84 (1957), 545-558.

6. _- On H-spaces and their homotopy groups, Quart. J. Math. Oxford Ser. (2) 11 (1960), 161-179.

7. — , Reduced product spaces, Ann. of Math. (2) 62 (1955), 170-197. 
8. J. Milnor, Construction of universal bundles. II, Ann. of Math. (2) 63 (1956), 430-436.

9. - Lectures on characteristic classes (mimeographed), Princeton, 1957.

10. J. C. Moore, The double suspension and p-primary components of the homotopy groups of spheres, Bol. Soc. Mat. Mexicana 1 (1956), 28-37.

11. Y. Nomura, On mapping sequences, Nagoya Math. J. 17 (1960), 111-145.

12. J. P. Serre, Groupes d'homotopie et classes de groupes abéliens, Ann. of Math. (2) 58 (1953), 285-294.

13. J. D. Stasheff, On homotopy Abelian H-spaces, Proc. Cambridge Philos. Soc. 57 (1961), 734-745.

14. M. Sugawara, A condition that a space is group-like, Math. J. Okayama Univ. 7 (1957), 123-149.

15. - On a condition that a space is an H-space, Math. J. Okayama Univ. 6 (1957), 109-129.

16. J. H. C. Whitehead, Combinatorial homotopy. I, Bull. Amer. Math. Soc. 55 (1949), 213-245.

Massachusetts Institute of Technology,

CAMbridge, MassachusetTS 